\title{
Migration et asile en Tunisie depuis 2011 : vers de nouvelles figures migratoires?
}

Migration and Asylum in Tunisia since 2011: Towards a New Migration Profile? Migración y asilo en Túnez desde 2011: ¿hacia un nuevo perfil de la migración?

\section{Hassan Boubakri}

\section{OpenEdition}

Journals

\section{Édition électronique}

URL : https://journals.openedition.org/remi/7371

DOI : $10.4000 /$ remi.7371

ISSN : $1777-5418$

Éditeur

Université de Poitiers

\section{Édition imprimée}

Date de publication : 1 décembre 2015

Pagination : 17-39

ISBN : 979-10-90426-26-9

ISSN : 0765-0752

\section{Référence électronique}

Hassan Boubakri, «Migration et asile en Tunisie depuis 2011 : vers de nouvelles figures

migratoires ? », Revue européenne des migrations internationales [En ligne], vol. 31 - n³ et 4| 2015, mis en ligne le 01 décembre 2018, consulté le 14 avril 2022. URL : http://journals.openedition.org/remi/ 7371 ; DOI : https://doi.org/10.4000/remi.7371 


\section{Migration et asile en Tunisie depuis 2011 : vers de nouvelles figures migratoires?}

\section{Hassan Boubakri'}

\section{Introduction}

À l'instar des autres pays du Maghreb, la Tunisie a vu sa fonction migratoire évoluer depuis les années 1990. Elle a d'abord toujours été, et le demeure encore, un pays de départ : 1,3 million deTunisiens vivent à l'étranger. Mais dès la fin du XXe siècle, des migrants subsahariens transitent par la Tunisie pour traverser la Méditerranée, en direction des côtes italiennes, dans des mouvements destinés à contourner les contrôles aux frontières extérieures de l'espace Schengen. À l'ouest du Maghreb, le Maroc se transforme à son tour en pays de transit en direction des côtes espagnoles (Boubakri, 2006a). Le Sud algérien sert également de carrefour migratoire en direction du Maroc et de la Libye, le plus grand pays de destination sur la rive méridionale et orientale de la Méditerranée depuis les années 1970. Jusqu'en 2010, des sources officielles libyennes ${ }^{2}$ situaient le nombre d'étrangers présents dans le pays à au moins 1,5 million de personnes, soit le quart de la population libyenne recensée en 2006. La majorité de ces migrants restaient dans le pays pour y travailler ou faisaient des allers-retours avec leurs pays d'origine limitrophes tels que l'Égypte, la Tunisie, le Soudan, le Tchad, le Niger ou le Mali, ou avec les pays plus lointains de la Corne de I'Afrique ou de l'Afrique occidentale. La partie restante de ces migrants a rejoint les réseaux de passages et de traversées en direction des côtes européennes. Les réfugiés fuyant les violences et les situations de guerre font de la Libye aussi un pays de transit.

La première décennie du XXle siècle a vu ces pays d'Afrique du Nord adopter des politiques de contrôle et de répression des migrants irréguliers, rejoignant ainsi celles suivies par les pays de la rive nord de la Méditerranée, faisant de l'Europe une zone de plus en plus inaccessible tant aux migrants économiques

1 Professeur de géographie à I'Université de Sousse, membre du laboratoire SYFACTE/ Université de Sfax, chercheur associé à I'IRMC, à Migrinter et à I'URMIS, Avenue Khalifa Karoui, Cité Sahloul 4, BP 526, 4000 Sousse-Sahloul,Tunisie ; hassan.boubakri@gmail. com

2 Ali Abdessalem Triki, secrétaire du Comité populaire général (ministre) à l'Union africaine, Associated Press, décembre 2003. 
qu'aux réfugiés et demandeurs d'asile. Le déclenchement de la révolution en Tunisie et de la rébellion libyenne en 2011, qui se sont soldées par le renversement des deux régimes, a radicalement changé la donne migratoire dans la zone (Boubakri, 2013a). Pays en guerre civile depuis cette date, la Libye est devenue peu sûre aussi bien pour les migrants que pour ses propres citoyens. Dès le début du conflit en février 2011, des travailleurs étrangers et des réfugiés vont quitter ce pays en direction des pays voisins, dont la Tunisie. Quelques semaines plus tard, ce sont les Libyens qui ont pris à leur tour la route de l'exil, en direction de laTunisie principalement.

Le voisinage entre la Tunisie et la Libye fait des deux pays, depuis 2011, un " tandem " migratoire particulier. La délicate transition politique de la Tunisie, d'un côté, qui a duré quatre ans (2011-2014) et, de l'autre, l'instabilité, suivie de la guerre civile et du chaos depuis 2011, font que l'on ne peut comprendre et analyser ces mouvements de populations, sans prendre en compte l'évolution du contexte migratoire et politique dans chacun des deux pays, comme dans la région. Beaucoup de changements ont affecté, depuis 2011, la nature et l'ampleur des flux de migrants et de réfugiés qui aboutissent en Tunisie et dans les pays tiers de la rive méditerranéenne ou les traversent; ils ont également affecté les routes et la manière dont les États en particulier, mais aussi les nouveaux acteurs (partis politiques, organisations de la société civile, organisations internationales), qui sont apparus après 2011, ont traité ces flux ou ont géré leur inscription dans les territoires et les sociétés ayant accueilli ces migrants.

Dans cet article, qui ne prétend pas à l'exhaustivité, l'analyse sera limitée à la manière dont la Tunisie accueille depuis cinq ans des réfugiés, sans avoir été avant 2011 un grand pays d'asile ou en contact direct avec des zones de guerre. L'intérêt de ce choix réside dans la variété des parcours, des origines et des statuts (ou le défaut de statut) de ces réfugiés. La première partie est consacrée à l'analyse du contexte qui a fait de laTunisie une terre d'exil pour les travailleurs étrangers, puis pour les Libyens, qui ont fui la guerre en 2011, et à la manière dont laTunisie a géré le premier camp de réfugiés sur son territoire. La deuxième partie est consacrée aux conditions de la circulation et du séjour en Tunisie de deux groupes de populations (Libyens et Syriens), dont les pays d'origine sont en guerre. L'intérêt de l'étude de ces deux groupes réside dans le fait qu'il s'agit de ressortissants de pays qui partagent avec la Tunisie une proximité culturelle liée à la communauté de langue, de religion et d'histoire. En outre, la Tunisie partage une frontière commune avec la Libye, deuxième pays d'immigration des travailleurs tunisiens, et d'anciennes traditions de circulation des personnes avec la Syrie qui n'a jamais imposé de visa auxTunisiens qui voulaient s'y rendre pour travailler ou étudier. Par conséquent, l'opinion publique et les autorités tunisiennes considèrent que ces populations "frères " sont " chez elles en Tunisie ". Mais sur le plan juridique et sécuritaire, elles sont exposées, au même titre que les autres étrangers, surtout ressortissants des pays tiers, à la rigidité de la législation tunisienne qui peut donner lieu à des abus de la part de l'administration ou des forces de sécurité. Fuyant leurs pays en guerre, elles sont rentrées en Tunisie à la recherche d'une protection et pour bénéficier des services publics auxquels elles n'ont plus accès dans leurs pays. Réfugiées en Tunisie, elles n'en ont pas le statut. Les Libyens, et même une partie des Syriens, ne déposent pas de demande d'asile, ou ne veulent pas le faire. En outre, les autorités tunisiennes ne souhaitent pas que les Libyens surtout, mais aussi les Syriens dans une 
moindre mesure, accèdent au statut de réfugiés afin d'accélérer leur retour dans leur pays d'origine dès la fin des conflits. Enfin, la circulation des Libyens et des Syriens sur le territoire tunisien, avec des chassés-croisés assez intenses, rend difficile la caractérisation de leur présence. Cet article a donc pour ambition de répondre, au moins partiellement, aux questions posées et d'éclairer la situation particulière de ces " étrangers " sans statuts ${ }^{3}$.

\section{Après 2011 : Tunisie, pays d'exil et d'asile}

LaTunisie a connu une expérience migratoire que l'on peut qualifier d'inédite en l'espace de trois mois (janvier à mars 2011) avec une succession de trois vagues migratoires, d'une ampleur inégalée dans son histoire ${ }^{4}$. La liberté de la presse et de la parole après la révolution ont permis de mettre au centre du débat public la question des migrations irrégulières des jeunes Tunisiens d'une part et, d'autre part, celles des populations subsahariennes " en transit ", un sujet jusque-là tabou et dont le traitement était strictement contrôlé par le régime de l'époque et jamais, ou très peu, abordé dans les médias (Boubakri, 2009).

\section{La crise de l'année 2011 : trois vagues migratoires successives}

\section{L'exil des travailleurs étrangers et des Libyens}

Dans les semaines qui ont suivi la chute du régime le 14 janvier 2011, des milliers de jeunes Tunisiens ont traversé les quatre-vingt miles de mer qui séparent les côtes tunisiennes de l'île italienne de Lampedusa. Leur nombre a atteint à la fin du premier trimestre 20 500, pour baisser rapidement à 4500 personnes au deuxième trimestre, 3500 au troisième et pas plus de quatrevingt-seize migrants à la fin du dernier trimestre (Frontex, 2012).

À partir du 17 février 2011, l'éclatement du conflit libyen a poussé des centaines de milliers de travailleurs étrangers (dont les Tunisiens) à se diriger vers la Tunisie en premier lieu. De mars à octobre 2011, 770000 travailleurs étrangers ont franchi les frontières terrestres libyennes en direction des pays voisins ${ }^{5}$, dont environ la moitié en direction de la Tunisie ${ }^{6}$. La quasi-totalité de ces exilés a été rapatriée en quelques semaines vers leurs pays d'origine, par voie aérienne pour la plupart d'entre eux ou par voie maritime pour une partie

3 Une partie du contenu de ce texte est tiré des résultats des observations de terrain, des recherches et des rapports que I'auteur a réalisés depuis 2011, ainsi que de ses publications (cf. références bibliographiques).

4 Les conséquences des insurrections, tunisienne puis libyenne, sur l'évolution des flux migratoires à l'échelle du Maghreb et de la Méditerranée ont été amplement traitées (Boubakri et Potot, 2011 et 2012 ; Fargues et Fandrich, 2012 ; Baba, 2013 ; Boubakri, 2013 ; Natter, 2014).

5 Dans un bilan publié début novembre 2011, I'OIM (Organisation Internationale des Migrations) avance le chiffre de 768372 travailleurs migrants ayant quitté la Libye depuis le début du conflit, dont 345238 sont entrés en Tunisie (soit $45 \%$ du total) et 242797 en Égypte. Le reste était réparti entre le Niger (84 428) et le Tchad (52 000). En outre, 136749 émigrés tunisiens ont regagné leur pays au cours de la même période (IOM, 2011).

6 Dans un bilan provisoire établi deux mois après le déclenchement de la guerre, il ressort que 179284 travailleurs étrangers ont traversé la frontière tunisienne en deux mois (mars-avril 2011). Les Égyptiens occupent le premier rang (85 850 migrants), suivis des Bangladeshis (27 780), des Soudanais (13 857), des Tchadiens (11 905), les Marocains (8 450), etc. (Chouat et Liteyem, 2011). 
des Égyptiens par exemple (Chouat et Liteyem, 2011 ; OIM, 2011). Seuls quelques milliers de réfugiés sont restés en Tunisie dans le camp de Choucha (voir infra) près de la frontière.

L'accueil des travailleurs étrangers avait été organisé conjointement par les nouvelles autorités tunisiennes et par des initiatives populaires (organisations d'une société civile naissante, mobilisation des populations locales, etc.) avant que n'intervienne l'aide internationale. Cette première expérience avait montré la liberté d'initiative retrouvée après des décennies de contrôle et de main mise par les régimes autoritaires successifs de Bourguiba puis de Ben Ali (Boubakri et Potot, 2011).

La troisième vague migratoire qu'a connue la Tunisie, en lien avec le conflit libyen, était composée de ressortissants libyens, des familles pour la plupart, qui s'étaient exilés chez le voisin occidental pour fuir les combats, l'insécurité, les pénuries et la désorganisation des services publics. La Tunisie n'est cependant pas à sa première expérience d'accueil des réfugiés libyens. Un siècle plus tôt, suite à l'invasion italienne de la Libye en 1911, des dizaines de milliers de familles libyennes étaient arrivées en Tunisie, au terme d'un dramatique exode de masse.

\section{Sociabilités tuniso-libyennes et conflits nés de l'exil}

Si pratiquement tous les travailleurs migrants exilés de Libye au début du conflit sont restés dans les camps aménagés près de la frontière pour faciliter leur rapatriement qui s'est fait à partir de l'aéroport de Djerba et du port commercial de Zarzis (Chouat et Liteyem, 2011), les exilés libyens se sont, eux, dispersés sur une large partie du territoire tunisien, mais en nombre supérieur dans la région du Sud et dans des villes littorales, telles que Zarzis, Djerba, Sfax, Sousse ou Tunis. La proximité géographique et culturelle entre les populations habitant de part et d'autre de la frontière a facilité l'accueil d'une partie des familles libyennes dans les foyers des familles tunisiennes transfrontalières, ce qui est à noter dans ces milieux sociaux plutôt conservateurs (Boubakri et Potot, 2012). Après la disparition de Kadhafi et l'arrêt provisoire du conflit à la fin de l'année 2011, une grande partie des Libyens présents en Tunisie sont rentrés dans leur pays, seuls restaient en Tunisie les proches de l'ancien régime qui craignaient pour leur vie ${ }^{7}$.

Cette arrivée d'exilés libyens en Tunisie a donné lieu dans un premier temps à des témoignages de sympathie et de solidarité réciproques entre Libyens et Tunisiens, d'autant plus que les deux peuples avaient eu le sentiment d'avoir réussi à se débarrasser de leurs régimes autoritaires ou dictatoriaux. Des formes de sociabilités se sont développées. On a, par exemple, assisté à des mariages mixtes et à des échanges de visites, mais la multiplication des milices et l'effondrement de l'autorité de l'État libyen dès 2011 ont ouvert la voie à des pratiques

7 Le 25 décembre 2012, Moustapha Abdeljalil, ex-président du Conseil National de Transition (CNT), déclarait " Nous ne devons pas oublier que la Tunisie accueille encore aujourd'hui environ 600000 Libyens, qui sont nos frères [...] " (El Maghrib,Tunis, 25 décembre 2012, p. 18). Ce chiffre qui paraît très important couvrait à l'époque deux catégories des Libyens présents en Tunisie : les proches de l'ancien régime qui craignaient de rentrer en Libye ; les personnes et les familles qui avaient préféré s'installer en Tunisie pour bénéficier de la relative stabilité du pays, alors que les pénuries et la désorganisation des services publics en Libye commençaient à se faire sentir dès 2011. 
mafieuses et à des abus divers dont étaient victimes, entre autres, les Tunisiens revenus en masse en Libye pour y travailler, ou ceux qui font des allers-retours en relation avec des activités de l'économie informelle transfrontalière. Les violences subies par les Tunisiens (enlèvements, séquestrations, braquages, disparitions ${ }^{8}$ et même, assassinats) ont contribué à détériorer les relations entre les deux populations. Des Libyens qui rentraient en Tunisie avaient été, de leur côté, victimes de représailles sur les routes du Sud tunisien en particulier. Des actions de représailles et de contre-représailles se sont succédé depuis et se sont même exacerbées après la reprise du conflit entre partisans des différentes milices et tribus depuis 2014 (voir infra).

\section{Les limites de l'expérience de la gestion de l'asile en Tunisie}

Les réfugiés et les demandeurs d'asile qui vivaient en Libye avant la guerre se sont exilés en Tunisie, comme les autres travailleurs migrants étrangers qui ont été rapatriés dans leur majorité, à la différence des réfugiés qui sont restés dans le camp de transit de Choucha, implanté à sept kilomètres du poste frontalier de Ras J'dir, (voir Figure 1), dans l'attente d'une réinstallation. Le programme "IGSR " (Initiative Globale de Solidarité pour la Réinstallation) a été mis en place par le Haut Commissariat aux Réfugiés (HCR) pour répondre aux besoins de ces personnes qui ne pouvaient pas retourner dans leurs pays d'origine en guerre. Un peu plus de 3500 réfugiés ont fini par être réinstallés : $80 \%$ ont été accueillis par quatre pays (53\% aux États-Unis, 15,7 \% en Norvège, $7 \%$ en Suède et $6,5 \%$ en Allemagne). Les $20 \%$ restants ont été répartis dans d'autres pays (UNHCR, 2013 ; Boubakri, 2013).

\section{Du camp de transit à la tentative d'insertion urbaine}

Les derniers chiffres avancés par le bureau du HCR en Tunisie (UNHCR, 2015) situent le nombre des réfugiés et des demandeurs d'asile à 980 personnes. II s'agit d'un chiffre en baisse par rapport aux années précédentes : 1157 réfugiés en 2014 et 1225 en 2013 (UNHCR, 2014 et 2013). Faut-il rappeler qu'avant 2011, le nombre moyen de réfugiés statutaires en Tunisie ne dépassait pas la centaine, un plafond fixé par les autorités tunisiennes d'alors qui voulaient, par cette mesure arbitraire et officieuse, donner l'image d'un pays hostile à l'accueil des réfugiés ? Jusqu'en 2014, les réfugiés originaires de certains pays d'Afrique occidentale (comme la Côte d'Ivoire) ou de la Corne de I'Afrique (Somalie, Érythrée et Éthiopie) étaient majoritaires. Beaucoup d'entre eux ont bénéficié de la réinstallation, ce qui explique la baisse de leur effectif en 2015, contre la montée de celui des Syriens qui, avec 629 réfugiés statutaires, occupent désormais la première place. Ils sont suivis par les Ivoiriens (127 personnes), puis les Soudanais (cinquante-trois personnes). 171 autres réfugiés appartiennent à vingt-et-un autres pays (UNHCR, 2015).

Si la plupart des réfugiés avec statut ont été réinstallés dans les années 20122013, le camp de Choucha comptait encore en juin 2013 environ 200 personnes appartenant à d'autres catégories. II y avait d'abord les " déboutés ", c'est-à-dire les demandeurs d'asile qui n'ont pas bénéficié du statut de réfugié. II y avait

8 Deux journalistes tunisiens ont été enlevés en septembre 2014 et sont toujours portés disparus. 


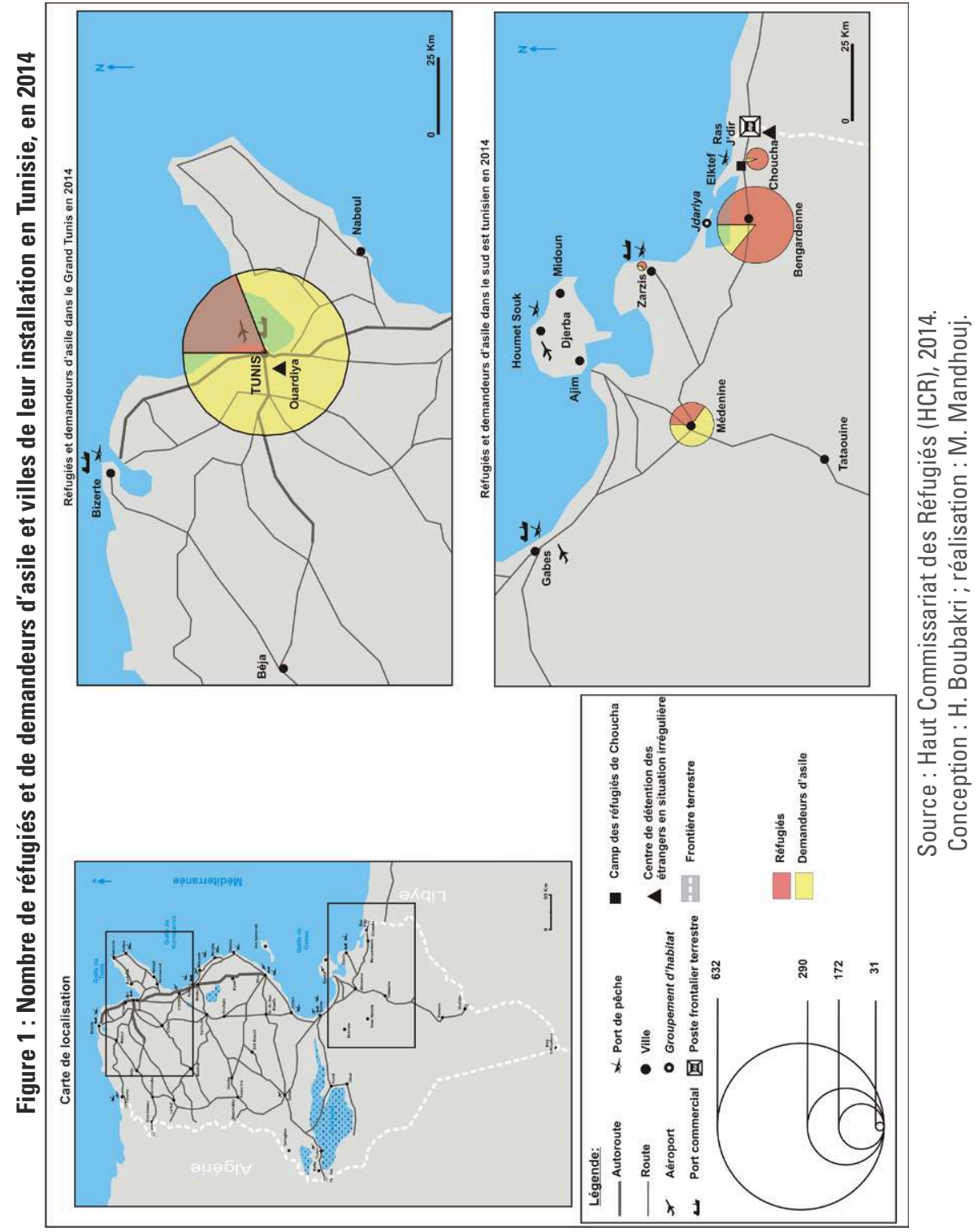


aussi les réfugiés appelés "statutaires ". Une partie de ces derniers étaient en attente de réinstallation alors que d'autres n'avaient pas reçu de promesse de réinstallation. Considérant que le nombre des réfugiés restés à Choucha ne justifiait plus le maintien de ce camp perdu entre les dunes de sable, le HCR avait décidé de le fermer au 30 juin 2013 et de proposer aux réfugiés un " programme d'intégration urbaine ${ }^{9}$ dans les villes voisines du Sud-Est (Bengardenne, Zarzis, Médenine) (cf. Figure 1). Si les réfugiés en attente d'installation ont accepté de bénéficier de ce programme, les "déboutés " et ceux qui n'avaient pas obtenu de promesse de réinstallation ont refusé cette option et ont préféré rester dans le camp dans des conditions de précarité et de vulnérabilité extrêmes (Boubakri, 2013) pour maintenir la pression sur le HCR et sur les autorités tunisiennes pour qu'ils règlent leur situation. Les uns, comme les autres, entretenant l'espoir de partir et de rejoindre ceux qui ont été réinstallés dans les pays du Nord.

Au cours des deux années qui ont suivi la fermeture officielle du camp de Choucha, des dizaines de réfugiés, parmi ceux qui ont refusé de le quitter, avaient dû se résoudre à compter sur eux-mêmes et à recourir aux services, risqués, des passeurs très entreprenants dans la zone transfrontalière et dans les villes-ports de l'Ouest de la Libye, dont Zuwara qui a toujours été, avant comme après 2011, la plateforme principale de départ des traversées en direction de Lampedusa et de Malte. Toutefois, une partie de ces réfugiés reste en Tunisie.

\section{Dispersion géographique et formes de sociabilités}

La présence des réfugiés et des demandeurs d'asile ne se limite plus àTunis, comme avant 2011, mais s'étend désormais à la zone frontalière avec la Libye. Avec 632 réfugiés en 2014, Tunis reste la première zone d'installation, mais les villes du Sud-Est (Bengardenne, Zarzis et Médenine) fixent près de la moitié $(48 \%)$ des réfugiés recensés dans le pays (cf. Figure 1), un nombre en baisse par rapport à celui de 2013 quand elles en fixaient 64,1\%. Cette tendance à la baisse du nombre de réfugiés dans la région - ils ne seraient plus que 200 à 300 en 2015, selon le responsable du bureau régional du Croissant RougeTunisien (CRT) à Médenine - est liée aux mouvements de départs vers la Libye qui touchent ces réfugiés. Nous assistons à une sorte de chassé-croisé entre les réfugiés qui arrivent en Tunisie et ceux qui la quittent en direction de la Libye. Grâce à la connaissance qu'ils ont acquise des lieux et des modes de circuler dans cette zone frontalière, une partie des occupants du camp de Choucha, qui continuent à refuser de le quitter, se transforment en intermédiaires entre, d'un côté, les passeurs et, de l'autre, les réfugiés et les migrants sauvés en mer et hébergés dans les villes de cette zone pour leur faire retraverser la frontière en direction des points d'embarquement en Libye, puis en direction des côtes italiennes et même en direction des côtes grecques, via la Turquie, par voie aérienne au départ de la Libye, puis par voie maritime à partir des côtes égéennes de la Turquie.

9 Ce programme est destiné, selon le HCR, à faciliter l'insertion en milieu urbain des réfugiés statutaires principalement : accès aux services essentiels comme les soins de santé et l'éducation, bénéfice d'une allocation, aide pour trouver un logement, cours de langue et de formation professionnelle, aide pour le lancement de microprojets (HCR, 2013). 
Point des départs irréguliers, cette zone frontalière du Sud-Est tunisien sert parfois aussi de base pour l'organisation des opérations de sauvetage en mer des embarcations qui partent des côtes libyennes notamment. En 2014, treize embarcations ont été secourues et 1400 migrants ont été débarqués dans les ports tunisiens d'el Ketef, J'dariya et Zarzis ${ }^{10}$ (cf. Figure1).

Progressivement, cette zone sert aussi d'espace où se croisent des migrants, des réfugiés, des passeurs et des trafiquants de migrants et même des djihadistes qui rejoignent les zones de guerre en Libye, en Syrie et en Irak. Des catégories auxquelles l'on peut ajouter les acteurs qui interviennent dans les réseaux de transactions et d'échanges liés à l'économie informelle transfrontalière. Les figures de l'Étranger (migrants et réfugiés de toute origine, passeurs, transfrontaliers, etc.) sont désormais bien présentes dans cette région frontalière et participent à une visibilité croissante et renouvelée de l'altérité proche (Libyens, Syriens) ou lointaine (Africains subsahariens, Pakistanais, etc.) dans le paysage social local. Des sociabilités se construisent, rapprochant les populations locales des migrants et des circulants étrangers. Les réfugiés et les migrants sauvés en mer, hébergés dans les villes de cette zone, ainsi que les réfugiés de Choucha, trouvent à s'employer dans certains secteurs de l'économie locale comme maçons, ouvriers du bâtiment, jardiniers et surtout manutentionnaires embauchés par les commerçants pour charger ou décharger les véhicules de transport de biens et produits échangés entre la Tunisie et la Libye. Cette zone frontalière reste donc très impliquée par les différents flux migratoires, réels ou même potentiels ${ }^{11}$.

Malgré le caractère localisé de l'expérience du camp de Choucha, celle-ci a été largement portée à la connaissance du public par la communauté scientifique, mais aussi par le truchement des réseaux sociaux et par les ONG, alors même que l'État tunisien est resté en retrait.

10 Ces opérations ne correspondaient cependant pas aux minima du standard international de secours humanitaire requis dans de telles circonstances (assistance sanitaire, identification des réfugiés et des demandeurs d'asile parmi les personnes rapatriées ou secourues, conseils juridiques, conditions d'hébergement dignes, etc.). Un centre d'hébergement a été aménagé à Bengardenne pour abriter une partie de ces rescapés de la mer, les identifier et aider ceux qui voudraient rentrer dans leurs pays. Le HCR et I'OIM ont également assisté les autorités tunisiennes dans I'aménagement et I'équipement d'un centre de rétention appelé "Centre d'hébergement et d'orientation " dans l'enceinte du poste de la garde nationale de Ras J'dir, à la frontière avec la Libye. II était prévu que ce centre soit le deuxième de ce type (en plus de celui d'Ouardiya, à la périphérie Sud deTunis) pour la détention des étrangers en situation irrégulière arrêtés à la frontière ou interceptés en mer. Mais les autorités ont préféré réserver cet espace à I'hébergement des forces de sécurité frontalières dont le nombre n'a pas cessé d'augmenter dans un contexte de tensions sécuritaires et de risques terroristes dans cette région voisine de la Libye. Les organisations internationales (OIM, HCR, Islamic Relief) et tunisiennes (le CRT) ont alors été amenées à aménager des locaux souvent inadaptés ou exigus à Bengardenne ou à Zarzis pour accueillir, héberger et identifier ces rescapés, ce qui prend souvent plusieurs semaines.

11 Durant I'été 2014, à la reprise des combats entre milices libyennes, le HCR, I'OIM et les autorités tunisiennes ont travaillé sur des scénarios d'exode massif des populations libyennes pour se mettre à l'abri des violences et ont programmé des dispositifs de secours d'urgence dans les villes frontalières avec la Libye (Bengardenne, Tataouine, Dhéhiba, etc.). Des personnels des deux organisations ont été réaffectés à Zarzis et à Tataouine pour être prêts à surveiller les rythmes d'arrivées des Libyens à la frontière et donner l'alerte en cas de besoin. 


\section{Les hésitations de l'État tunisien}

LaTunisie a ratifié la Convention de Genève de 1951 ainsi que son protocole annexe de 1967. Elle est également signataire de la Convention de 1969 de l'Organisation de I'Union africaine (UA) relative aux réfugiés. Elle n'a toutefois pas une loi interne sur l'asile. La Tunisie délègue encore la reconnaissance du statut de réfugiés sur son territoire à la représentation du HCR dans le pays. Les travaux préparatoires d'un projet de loi sur l'asile datent de 2011. Bien que prêt depuis 2014, ce projet n'est toujours pas soumis au parlement. Les autorités tunisiennes hésitent à promulguer cette loi de crainte de se retrouver liées par des obligations auxquelles elles ne peuvent répondre : mise en place de structures de détermination du statut de réfugiés, accueil, assistance des demandeurs d'asile, intégration des réfugiés reconnus, gestion des recours, modification de la législation relative aux migrants et aux étrangers, etc. (REMDH et CeTuMA, 2015). En effet, les lois tunisiennes sont muettes sur la définition des différentes catégories de migrants : "Aucun texte ne définit les notions de "migrant", "d'immigrés" ou de "réfugiés". II convient alors de s'orienter vers les textes d'origine internationale "(Ben Achour et Ben Jémia, 2011). D’un autre côté, les conflits ethniques et les guerres civiles que connaissent des pays de régions limitrophes (Libye, pays du Sahel, Syrie, etc.) mettent sur les routes de l'exil un grand nombre de réfugiés. La Tunisie est l'un des pays de transit de ces populations en direction de I'Union européenne (UE). Les autorités tunisiennes mettent en avant le risque de devoir accueillir un nombre croissant de réfugiés en cas d'exacerbation des conflits dans son voisinage immédiat - la Libye - et ceux qui sont plus lointains, comme les conflits syriens ou irakiens. En effet, pour faire face à la crise migratoire en Méditerranée provoquée par ces conflits depuis 2011 et surtout depuis 2014, les pays de l'Union européenne cherchent à renforcer la surveillance des frontières extérieures de I'UE et à externaliser l'accueil et l'assistance portés aux réfugiés vers les pays tiers méditerranéens comme la Turquie, le Liban, la Jordanie ou la Tunisie.

Depuis cinq ans, la Tunisie connaît une autre expérience, liée à l'accueil de ressortissants de pays en guerre : les Libyens et les Syriens. Contrairement aux réfugiés du camp de Choucha, en majorité originaires des pays du Sahel, la présence des Libyens, et dans une moindre mesure des Syriens, se caractérise par une large répartition sur le territoire tunisien et par une grande mobilité ${ }^{12}$.

\section{Circulation et séjour des Libyens et des Syriens en Tunisie}

\section{Les limites de I'outil statistique dans l'estimation de la mobilité des personnes}

Les statistiques sur les flux migratoires entre la Tunisie et l'étranger souffrent d'un manque de données. II concerne d'abord la migration des Tunisiens au

12 Les chercheurs commencent à peine à s'intéresser à ces populations et aux sociabilités qu'elles développent en Tunisie. Mais il est difficile de connaître leur nombre et la statistique publique tunisienne n'arrive pas à combler les déficits de données et les lacunes dans le dénombrement des ressortissants étrangers quand ils résident en Tunisie, ou lorsqu'ils traversent les frontières. 
départ, ou au retour de l'étranger. Il concerne aussi les étrangers qui s'installent en Tunisie pour des durées plus longues que ne leur permet pas la législation locale. L'Institut National de la Statistique (INS) a recensé 53490 étrangers résidents en Tunisie en 2014 (voirTableau 1), contre 28100 en 2004 (INS, 2004 et 2015). Ce chiffre de 2014 est en deçà des faits. En effet, I'INS ne dénombre que 8772 Libyens et 1024 Syriens. Or, le conflit libyen par exemple, avec ses péripéties (la guerre de 2011, la transition fragile des années 2012-2013, puis la reprise de la guerre civile et le chaos qui s'ensuivit depuis 2014) a donné lieu à l'entrée et l'installation d'un grand nombre de Libyens ${ }^{13}$. Les données de I'INS sont très loin de prendre en considération ces situations.

Tableau 1 : Effectifs des étrangers recensés en Tunisie en 2014

\begin{tabular}{|c|c|c|c|c|c|}
\hline \multicolumn{2}{|c|}{$\begin{array}{l}\text { Ressortissants des } \\
\text { pays arabes }\end{array}$} & \multicolumn{2}{|c|}{$\begin{array}{l}\text { Ressortissants des pays } \\
\text { d'Afrique subsaharienne }\end{array}$} & \multicolumn{2}{|c|}{$\begin{array}{c}\text { Ressortissants des pays } \\
\text { européens, d'Amériques et des } \\
\text { autres pays }\end{array}$} \\
\hline Algériens & 9996 & Maliens & 958 & Français & 8284 \\
\hline Libyens & 8772 & Camerounais & 689 & Italiens & 2118 \\
\hline Marocains & 5565 & Ivoiriens & 607 & Allemands & 1393 \\
\hline Mauritaniens & 508 & Nigérians & 522 & Autres Européens & 3212 \\
\hline Égyptiens & 1093 & Sénégalais & 394 & Américains & 584 \\
\hline Syriens & 1024 & Autres & 4354 & Autres pays & 1895 \\
\hline Irakiens & 550 & & & & \\
\hline Palestiniens & 494 & & & & \\
\hline Autres & 478 & & & & \\
\hline \multirow{2}{*}{ Total } & 28480 & \multirow{2}{*}{ Total } & 7524 & \multirow{2}{*}{ Total } & 17486 \\
\hline & $53,2 \%$ & & $14,1 \%$ & & $32,7 \%$ \\
\hline \multicolumn{3}{|c|}{ Ensemble } & \multicolumn{3}{|c|}{53490} \\
\hline
\end{tabular}

Source : Institut National de la Statistique, RGPH, 2014.

Les soldes aux frontières sont dans ce cas des indicateurs qui peuvent nous permettre de corriger, au moins partiellement, ces écarts. En cinq ans (20092013), 1,8 million de Libyens, en moyenne par an, sont rentrés en Tunisie, alors que la moyenne des sorties n'a pas dépassé 1,5 million de personnes par an (INS, 2010 et 2014) (voir Figure 2). Leur solde positif a plus que triplé (+ $250 \%$ ) en I'espace de cinq ans, passant de 194000 en 2009 à 677000 en 2013. L'écart entre les entrées et les sorties ne cesse de s'élargir, montrant ainsi que le nombre des Libyens qui choisissent de prolonger leur séjour en Tunisie ne cesse donc de croître. Viennent s'ajouter à ce premier contingent les proches de l'ancien régime qui, craignant pour leur sécurité s'ils traversent les frontières, sont forcés de rester enTunisie. Ces populations ne sont pas comptabilisées. L'INS n'a

13 La Mission d'appui des Nations Unies en Libye a lancé le 10 décembre 2015 un appel pour une aide d'urgence de 165 millions de dollars en faveur de 2,4 millions de Libyens pour répondre aux besoins dans les domaines des services de santé (vaccins, médicaments, accès au centre de soins, accouchements sous surveillance médicale), de l'alimentation, de la protection, de l'eau potable, de l'assainissement, de la lutte contre les maladies infectieuses, etc. "II existe plus de 2 millions de personnes qui ont besoin d'assistance, dont les affamés, les malades, les personnes privées de protection, les réfugiés, ceux qui ont eu à subir des violences physiques ou psychologiques, les femmes victimes de violences, ainsi que les enfants et les jeunes privés de leur école " (chef de la Mission d'appui des Nations Unies en Libye ; http://www.alwasat.ly/ar/news/libya/88874). 
recensé que les étrangers qui déclarent être résidents réguliers en Tunisie et n'a pas pris en compte la présence d'un grand nombre d'autres étrangers qui ont été contraints de quitter leurs pays d'origine et de s'installer en Tunisie dans l'attente de la résolution des conflits qui déchirent certains pays (réfugiés syriens, ressortissants libyens, mais aussi ressortissants de quelques pays subsahariens tels que le Mali, le Nigeria, la République centrafricaine, la Somalie, etc.).

Figure 2 : Mouvements (entrées et sorties, en milliers) des Libyens aux frontières tunisiennes entre 2009 et 2013 (tous postes frontaliers confondus)

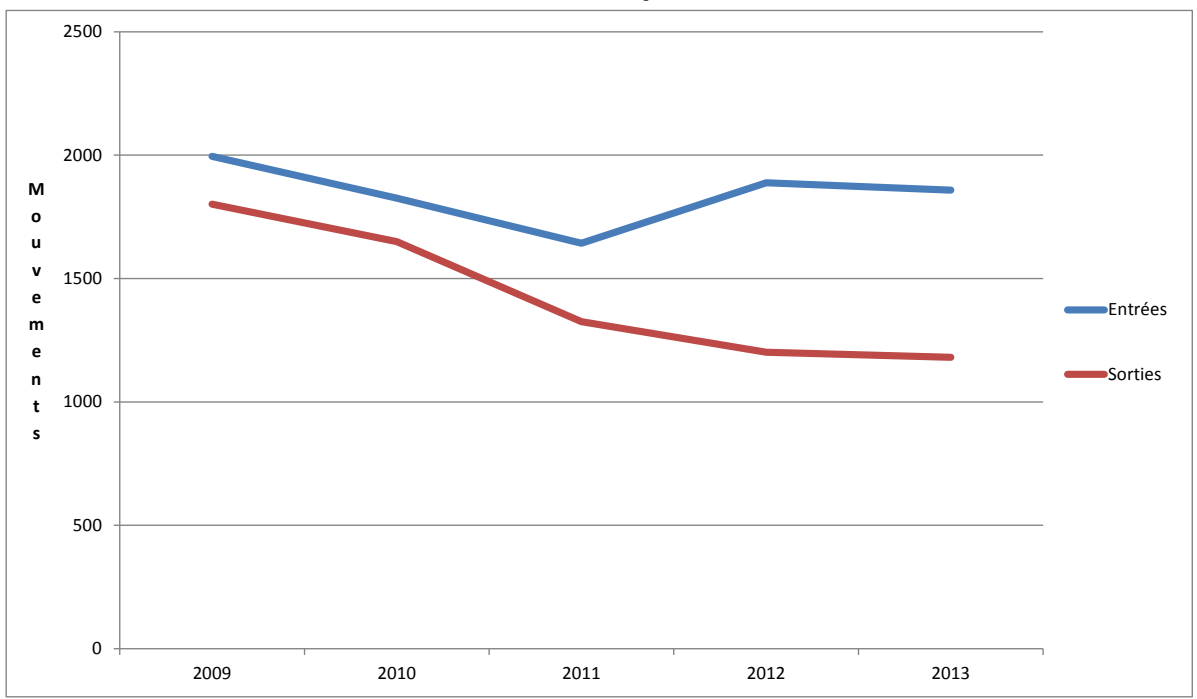

Source : Institut National de la Statistique, RGPH, 2014.

\section{Les Libyens en Tunisie, quelle catégorie : réfugiés, résidents temporaires ou touristes?}

En 2011, 100000 familles libyennes se sont réfugiées en Tunisie. Après le retour de la majorité dès la mort de Kadhafi en octobre 2011, seules sont restées en Tunisie les personnes alliées ou proches de l'ancien régime. Elles ont constitué le premier noyau d'un contingent de ressortissants libyens qui n'a cessé de grandir depuis 2012, conséquence de la désorganisation des services publics, de la détérioration des infrastructures et surtout de la reprise de la guerre entre milices et factions depuis le début de I'année $2014^{14}$.

14 Le séjour prolongé en Tunisie des ressortissants libyens fait l'objet depuis deux ans de questionnements récurrents. Les médias, les responsables politiques, les syndicats, les associations et même les organisations internationales avancent des chiffres contradictoires qui vont de quelques centaines de milliers à 2 millions. L'OIM écrit sur son site "Suite à la crise libyenne, entre 150000 et 2 millions de Libyens se sont installés en Tunisie depuis 2011 [...] » (OIM, 2015). L'OIM et L'ONM (Observatoire National de la Migration) se sont engagés dans une étude intitulée "Étudier les besoins et l'impact socio-économique des Libyens en Tunisie " et dont les résultats seront connus prochainement (Cf. http://tunisia.iom.int/news/etudier-les-besoins-et-I\%E2\%80\%99impact-socio\%C3\%A9conomiques-de-la-pr\%C3\%A9sence-libyenne-en-tunisie). 


\section{Asile, séjour ou circulation ?}

Il y a en Tunisie une confusion entre, d'un côté, la mobilité ou la circulation des ressortissants libyens entre leur pays et la Tunisie et, de l'autre, leur séjour prolongé, celui des familles notamment. L'absence de toute autorité et la défaillance de la plupart des services publics (santé, éducation, justice, réseaux électriques, d'eau potable et de communications, etc.) poussent de plus en plus de Libyens à rejoindre la Tunisie en attendant des jours meilleurs : hauts responsables de l'ancien régime, commerçants, familles des membres des milices armées et des militaires combattant dans les rangs de l'armée contrôlée par le gouvernement de Toubrouk ${ }^{15}$, journalistes, fonctionnaires, universitaires, juges et membres des ONG actives dans le domaine des droits de I'homme ; l'ensemble de ces personnes craignant pour leur vie. Comment les nommer? Réfugiés ? Résidents temporaires ? Frontaliers ? Touristes ? Ou alors assiste-t-on à l'émergence d'une nouvelle catégorie?

Ni les ressortissants libyens, ni leur État, ni les autorités tunisiennes n'utilisent les termes d'" asile " ou de "réfugiés ". D'ailleurs, selon le bureau du HCR à Tunis, les demandes du statut de réfugiés déposées par les Libyens se comptent sur les doigts de la main. L'insécurité et la menace terroriste contribuent à cette confusion des catégories et au raidissement des positions des autorités tunisiennes. En effet, après I'attentat du 24 novembre 2015 sur I'avenue Mohamed V àTunis contre la garde présidentielle, laTunisie a fermé les frontières avec la Libye pour une durée de deux semaines ${ }^{16}$. C'est la première mesure de ce type depuis vingt-huit ans, quand les frontières ont été rouvertes en 1988 et la libre circulation instaurée au profit des ressortissants des deux pays par la suppression des visas.

Le chiffre avancé par I'INS de 8772 Libyens résidents en Tunisie a provoqué une réaction très vive du département des études de l'Union GénéraleTunisienne du Travail (UGTT), principal syndicat du pays, qui a contesté cette statistique en estimant à son tour qu'il y avait une " sous-estimation flagrante " de la présence des ressortissants libyens dans le pays. Les protestations de I'UGTT sont à mettre en lien avec le contexte social et économique de laTunisie, caractérisé par la détérioration des principaux indicateurs économiques et sociaux depuis 2011.

\section{Les effets économiques du séjour des Libyens en Tunisie}

Depuis le début de la guerre civile en Libye en 2011, la pénurie des produits et services de base n'a cessé de s'amplifier, entrainant une demande croissante et des poussées inflationnistes sur le marché tunisien, dénoncées notamment par I'UGTT. Ce syndicat insiste sur l'impact de la présence "des frères libyens " ${ }^{17}$ sur I'inflation et, plus particulièrement, sur les charges qui pèsent sur les services

15 Deux gouvernements antagonistes se disputent la légitimité et la reconnaissance internationale. Le premier est celui de Toubrouk (Cyrénaïque), issu de la Chambre des représentants et reconnu par la communauté internationale, mais qui ne contrôle qu'une partie de la Cyrénaïque. Le deuxième est celui de Tripoli, issu du Congrès national, I'exparlement, mais qui ne contrôle qu'une partie de la Tripolitaine, dont la capitale.

16 Seuls ont été autorisés les retours des Tunisiens et des Libyens dans leurs pays respectifs.

17 Terme utilisé couramment en Tunisie, qui masque les incompréhensions, les malentendus, voire les tensions entre Tunisiens et Libyens. 
publics (réseaux de distribution de l'eau potable et de l'électricité, services de santé), ou encore sur la hausse de la consommation des produits alimentaires et pétroliers. Ces services et ces produits sont subventionnés par l'État à travers la Caisse Générale de Compensation (CGC) préservant ainsi le pouvoir d'achat des classes moyennes et populaires. Mais la demande liée à la présence prolongée des Libyens a conduit à une augmentation des prix sur le marché tunisien et a forcé les pouvoirs publics à accroître la subvention consacrée à la CGC (voir Tableau 2), ce qui se traduit par le creusement du déficit du budget de l'État.

Tableau 2 : Évolution des montants (en MDT : Millions de Dinars Tunisiens) consacrés à la Caisse Générale de Compensation (CGC)

\begin{tabular}{|l|l|l|l|l|l|l|}
\hline Année & 2009 & 2010 & 2011 & 2012 & 2013 & 2014 \\
\hline Montant & $800,0^{*}$ & $1500^{* *}$ & $2869,2^{* *}$ & $3624,1^{* *}$ & $5514,0^{* *}$ & $4154,0^{* *}$ \\
\hline
\end{tabular}

Cette situation a parfois poussé les autorités tunisiennes à intervenir pour interdire provisoirement l'exportation des produits subventionnés (lait, céréales, huiles végétales) ou non (certains légumes) vers la Libye, ce qui a ouvert la voie aux réseaux de contrebande transfrontalière pour répondre aux besoins du marché libyen. La pénurie de certains produits alimentaires avait même touché la Tunisie en 2011 et en 2014.

Depuis 2014, l'instauration d'une zone militaire d'exclusion le long de la frontière terrestre avec la Libye, rend de plus en plus difficile la contrebande de ces produits ${ }^{18}$. Suite à l'attentat de Sousse en juin 2015, les autorités ont entrepris la construction d'un mur de sable, doublé d'un fossé sur une distance de 200 kilomètres le long de cette même frontière. Si certains articles et interventions dans les médias ou des discours des hommes politiques mettent l'accent sur la " menace " que représente la présence des Libyens en Tunisie, quelques voix cependant soulignent la contribution " décisive " des ressortissants libyens (et algériens) à l'économie du tourisme depuis 2011 et notamment en 2015, à la suite de l'effondrement du nombre des touristes européens après les attentats du Bardo, de Sousse, puis de Tunis ${ }^{19}$.

\footnotetext{
18 Les services de douane, les forces de sécurité, ainsi que des unités de l'armée, chargés de la surveillance commune de la zone frontalière, font régulièrement état d'opérations de saisie des marchandises et des produits de la contrebande (Cf. www.assabahnews.tn).

19 Les dépenses moyennes des ressortissants libyens (ou algériens) représentent trois à quatre fois celles des Européens ; les dépenses des touristes maghrébins sont directement injectées dans l'économie locale (hébergement, consommation, restauration, commerces et autres services), ce qui n'est pas le cas des Européens, les dépenses transitant par les tours opérateurs.
} 


\section{Les conditions de circulation et de séjour des Libyens en Tunisie}

Comme il a été signalé plus haut, c'est grâce à l'instauration de la libre circulation des ressortissants des deux pays depuis 1988 que les mouvements transfrontaliers se sont intensifiés. La seule contrainte, réglementaire, réside dans la nécessité de ne pas dépasser la durée légale de résidence de trois mois pour tout ressortissant étranger $\mathrm{s}^{\prime} i \mathrm{i}^{\prime}$ 'est pas résident régulier ${ }^{20}$. Pour être en règle vis-à-vis des services de l'immigration, les Libyens doivent sortir deTunisie pour renouveler leur séjour touristique.

Mais l'exacerbation de la guerre civile en Libye et les risques qu'elle génère sur la sécurité des personnes (meurtres, enlèvements, disparitions, abus divers) poussent de plus en plus de Libyens à limiter ou à reporter leurs retours, ce qui se traduit par le prolongement de leur séjour en Tunisie. Le ministère tunisien de I'Intérieur tolérait cette situation et n'appliquait pas les sanctions prévues par la loi. Mais suite à l'attentat de Tunis du 24 novembre 2015, les autorités tunisiennes ont déclaré la «Mise à jour du registre des étrangers établis en Tunisie ${ }^{21}$. Les effets de ces mesures sont difficilement prévisibles, mais on peut $\mathrm{s}^{\prime}$ attendre à un durcissement du traitement de la présence étrangère avec des contrôles accrus, des mesures d'expulsion et éventuellement de détention aux dépens des ressortissants libyens ${ }^{22}$, de ceux des pays subsahariens, et même aux dépens des réfugiés et des demandeurs d'asile tels que les Syriens.

\section{Baisse du pouvoir d'achat et difficultés d'accès aux services de santé}

L'usage des infrastructures médicales tunisiennes était fréquent pour les étrangers et particulièrement pour les Libyens bien avant 2011, mais après cette date la Tunisie est devenue la destination principale des patients et des blessés de guerre libyens. De 2011 à 2013, I'État libyen prenait en charge l'intégralité des frais médicaux de ses ressortissants, mais la reprise du conflit a profondément affecté les capacités de paiement des deux gouvernements antagonistes de Tripoli à l'Ouest et de Toubrouk dans l'Est. La désorganisation du système bancaire libyen et la crise de liquidités jouent sur les capacités de consommation et de dépenses qui ne cessent de diminuer. Ces difficultés ont amené un grand nombre d'entre eux à rechercher, depuis 2014, des logements à loyers modérés dans les quartiers populaires de l'Ouest et du Sud de Tunis (Ibn Khaldoun, Omrane supérieur ou Hammam Lif, etc.), alors que, dans les années 2011 à 2013, la plupart se logeaient dans les quartiers riches au nord de la capitale (Ennasr, Berges du Lac, Soukra et Laouina).

20 Le bénéfice d'une carte de séjour temporaire (valable une année) ou ordinaire (valable deux ans) ou " longue durée " (valable dix ans) est conditionné par l'obtention d'un visa de séjour ou d'une autorisation de travail et par le bénéfice du regroupement familial (REMDH et CeTuMA, 2015).

21 Assabahnews (wwww.assabahnews.tn) et Tunis Afrique Presse (www.tap.info.tn/), 2015.

22 Les Libyens dont le séjour a dépassé trois mois en Tunisie ont été appelés à se faire enregistrer auprès de leurs consulats en Tunisie pour obtenir une carte consulaire qui devrait accompagner leurs passeports lors des contrôles de sécurité par les autorités tunisiennes. Cf. La Presse de Tunisie (2015) Les mesures du Conseil supérieur de la sécurité nationale, [en ligne], consulté le 05/01/2016. URL : http://www.lapresse. tn/06122015/106959/les-mesures-du-conseil-superieur-de-la-securite-nationale.html 
Depuis 2014, ils sont nombreux à vendre leurs biens (terrains achetés en Tunisie, or, voitures, etc.) pour faire face aux frais de logement et de santé essentiellement. Les résidents libyens ne sont désormais plus en état de se soigner dans le système de santé privé tunisien, comme ils le faisaient en 2011 et 2012. Des catégories de Libyens (personnes âgées, personnes atteintes de maladies chroniques ${ }^{23}$, enfants et mineurs, femmes enceintes, etc.) sont devenues encore plus vulnérables. Le directeur général d'une clinique privée de Tunis déclare :

"Les Libyens n'ont plus les moyens de régler les factures de soins, certains sont obligés de vendre leurs biens. [...] Non seulement le budget alloué par Tripoli depuis 2011 pour soigner certaines victimes des conflits se réduit de façon drastique depuis six mois, mais ceux qui disposaient d'assurances personnelles s'appauvrissent aussi... et le secteur médical avec eux. En 2014, la baisse du taux d'occupation a atteint jusqu'à $50 \%$ dans certaines cliniques privées où les Libyens, premiers touristes médicaux en Tunisie, représentent entre 50 et $70 \%$ des patients [...] ${ }^{24}$.

Les polycliniques privées tunisiennes n'acceptent plus les dossiers de prise en charge des ressortissants libyens. Elles exigent désormais d'être payées en espèces.

\section{Les nouvelles écoles libyennes en Tunisie}

La question de la scolarisation des élèves libyens s'est posée dès 2012 : des familles craignaient de rentrer chez eux ou le dysfonctionnement du système scolaire en Libye conduisait d'autres familles à rester en Tunisie, faute de pouvoir inscrire leurs enfants dans leurs villes ou régions d'origine. Les élèves libyens du primaire et du secondaire sont, dans ces conditions, restés privés d'école en raison de l'impréparation et l'incapacité du système scolaire tunisien à absorber un aussi grand nombre de ces élèves. À la demande des parents, l'ambassade de Libye àTunis va prendre en charge la construction ou la location de locaux pour ouvrir les premières unités scolaires libyennes autorisées par l'État tunisien. Cinq écoles publiques, financées par l'État libyen, ont été créées à Tunis, Hammamet, Sousse, Sfax et Mahdia. Deux autres écoles privées ont été créées dans des quartiers riches de Tunis (la Soukra et les Berges du Lac). En 2014, 1500 élèves sont inscrits dans le primaire et 400 dans le secondaire ${ }^{25}$.

Les écoles sont en sureffectif, le nombre moyen d'élèves variant entre trentecinq et quarante par classe, parfois même quarante-cinq, alors qu'avant 2014, la moyenne ne dépassait pas une quinzaine d'élèves par classe. Le manque de locaux a conduit les responsables de ces écoles à alterner les deux niveaux scolaires dans la même journée : la matinée est réservée aux classes de collège et de lycée, alors que les après-midi sont réservés aux classes du primaire. Cette expérience précoce fait face à des difficultés liées à la fragilité de la situation libyenne : retard de paiement des salaires du personnel libyen, déficit en enseignants, manque d'entretien des locaux, etc.

\footnotetext{
23 Une partie de ceux qui souffrent d'insuffisance rénale par exemple va désormais en Égypte où les dialyses sont moins onéreuses qu'en Tunisie.

24 Le Point (2015) Les Libyens en Tunisie : "chez eux " pour combien de temps ?, [en ligne], consulté le 05/01/2016. URL : http://afrique.lepoint.fr/economie/societe-les-libyensen-tunisie-chez-eux-pour-combien-de-temps-02-03-2015-1908957_2258.php

25 C'est dire que cette réponse aux besoins n'est qu'une goutte d'eau dans l'océan, surtout depuis la reprise de la guerre civile au début de I'année 2014.
} 
Beaucoup moins nombreux que les Libyens, la présence des réfugiés syriens est plus discrète, mais révèle aussi un manque de préparation des différents acteurs pour répondre à ce type de crises migratoires.

\section{L'exil des Syriens en Tunisie}

Le HCR avait recensé, en 2014, 3,5 millions de réfugiés syriens dans les pays du Moyen-Orient surtout. La part des pays d'Afrique du Nord est infime : 155000 réfugiés, soit $4,4 \%$ du total. Neuf de ces réfugiés sur dix (soit 140000 ) ont été dénombrés dans la seule Égypte (HCR, 2015). Les quatre pays du Maghreb (Maroc, Algérie, Tunisie et Libye) se partagent les 15000 restants, soit à peine 4000 par pays. Ce chiffre est confirmé par une source officielle tunisienne : le secrétaire d'État à la migration et à l'intégration sociale estime le nombre des Syriens présents en Tunisie à environ 4000 réfugiés ${ }^{26}$ (Assabah, 2015). Comme il a été mentionné plus haut, le bureau du HCR en Tunisie fait état de 629 réfugiés syriens inscrits auprès du bureau au 31 juillet 2015 (UNHCR, 2015).

\section{Un asile temporaire}

Avant 2011, ni la Syrie, ni la Tunisie n'imposaient de visa à l'entrée de leurs ressortissants sur leurs territoires respectifs. Mais le gouvernement tunisien a rompu en $2012^{27}$ ses relations avec la Syrie en signe de " [...] solidarité avec le peuple syrien dans ses revendications pour la liberté et la dignité $[\ldots]{ }^{28}$. L'une des conséquences de cette initiative tunisienne a été l'arrêt des liaisons directes entre les deux pays. Pour atteindre laTunisie, les réfugiés syriens qui cherchaient I'asile dans les années 2012 et 2013 étaient contraints de transiter par voie aérienne par l'Algérie, qui a toujours maintenu ses relations avec la Syrie, puis $d^{\prime}$ entrer en Tunisie, irrégulièrement, par les frontières terrestres.

La rupture des relations entre la Tunisie et la Syrie a fragilisé la situation des réfugiés syriens vis-à-vis de la loi tunisienne. Ils sont considérés, et traités, comme étant irréguliers. Ils font face à des contrôles fréquents, à des arrestations, des détentions prolongées et à des amendes pour séjour irrégulier. Les réseaux de trafic de migrants recrutent ces réfugiés en Tunisie et les conduisent en Libye, sans passer par les postes frontaliers tunisiens officiels. De Libye, ils sont embarqués en direction des îles italiennes (notamment Lampedusa).

La Tunisie est considérée comme un espace de transit, une terre d'asile temporaire pour tous les réfugiés et les demandeurs d'asile étudiés : les Syriens, autant que les Subsahariens d'ailleurs, veulent pratiquement tous quitter la Tunisie vers les pays européens, soit par le dépôt de leurs dossiers auprès du HCR, soit, irrégulièrement, par l'intermédiaire des passeurs. Après avoir presque tout perdu dans leur pays (membres des familles, maisons, terres, voitures, épargne, etc.), les réfugiés syriens déclarent avoir " coupé définitivement " avec

26 Assabah (2015) Déclaration du Secrétaire d'État à la Migration et à l'intégration Sociale, 16/09/2015, [en ligne], consulté le 05/01/2016. URL : www.assabah.com.tn

27 À l'époque sous le règne d'une coalition gouvernementale tripartite (appelée "Troïka ») dominée par le parti islamiste Ennahdha.

28 Turess (2012) L'organisation de la conférence sur la Syrie exprime la solidarité avec le peuple syrien (R. Abdessalem), [en ligne], consulté le 05/01/2016. URL: http://www.turess. com/fr/tapfr/119234 
leur pays et ne voient pas de perspectives de retour. Seule une petite minorité envisage de s'installer en Tunisie grâce aux réseaux de parenté ou de solidarité dont elle y dispose.

\section{La précarité des conditions d'accueil}

L'absence de dispositifs publics dédiés à l'accueil et à l'assistance aux réfugiés expose ceux qui se retrouvent en Tunisie à des conditions de vulnérabilité prononcée. Comme c'est le cas pour les Libyens, la scolarisation des enfants syriens pose problème du fait du nombre limité de places qui leur sont destinées dans les écoles publiques ${ }^{29}$. À cela, s'ajoute la difficulté de l'apprentissage de la langue française enseignée dès la troisième année du primaire en Tunisie, langue peu ou pas enseignée dans les écoles libyennes et syriennes. Les frais de scolarité ne sont pas accessibles à la majorité des familles de réfugiés syriens, dont une partie préfère envoyer leurs enfants mendier dans les rues ou autour des mosquées.

L'accès des Syriens aux services publics de santé est très fluctuant en raison de la multiplicité des intervenants, qui ne sont pas tous en relation les uns avec les autres, ce qui peut conduire à des interférences entre leurs actions : le HCR prend souvent en charge les frais médicaux des réfugiés à qui il a délivré le statut ; les hôpitaux publics admettent parfois ces réfugiés dans les services d'urgence ou pour la prise en charge des frais d'hospitalisation, d'accouchement ou pour des opérations chirurgicales; le ministère de la Santé intervient lui-même auprès de ces hôpitaux pour l'admission des patients réfugiés ; les organisations humanitaires (HCR, Médecins du Monde, Croissant Rouge Tunisien) participent aussi à la prise en charge des frais médicaux de ces réfugiés dans les hôpitaux publics ou auprès des cliniques privées.

Des actions de solidarité envers ces réfugiés se développent, venant des Syriens installés en Tunisie avant 2011 ou de la population tunisienne. Des médecins tunisiens ne font pas toujours payer les réfugiés ou les soignent à moindre coût. D'autres réfugiés encore bénéficient d'une indemnité de 250 DT par mois sur une durée de trois à six mois, octroyée par le gouvernement suisse à travers le HCR, mais ce montant est loin de pouvoir répondre à leurs besoins vitaux.

\section{Conclusion}

Les bouleversements politiques et sociaux survenus depuis 2011 sur la rive sud et est de la Méditerranée et dans le Sahel ont joué des rôles déterminants dans l'évolution des flux migratoires et dans celle des catégories de migrants qui quittent les pays de la région, les traversent ou s'y arrêtent. LaTunisie accueille sur son sol trois groupes de populations qui ont fui leur pays en guerre : les Syriens et les ressortissants de pays d'Afrique subsaharienne en guerre sont clairement désignés comme étant des réfugiés, alors que les Libyens, fuyant aussi leur pays livré aux violences et au chaos, affluent en Tunisie sans être considérés comme des réfugiés.

29 Quelques actions très symboliques d'insertion d'élèves syriens ou libyens dans les écoles tunisiennes ont été annoncées, mais ces réponses sont restées largement en deçà des besoins. 
Les Libyens qui rentrent en Tunisie ne cherchent pas, dans leur très grande majorité, à repartir vers l'Europe de manière irrégulière. Ceux qui cherchent à émigrer en Europe ou dans d'autres pays riches le font souvent en passant par les procédures de visas d'entrée dans ces pays. La Tunisie est désormais un pays de transit non seulement pour les migrants économiques en provenance d'Afrique subsaharienne notamment, mais aussi pour les réfugiés syriens et subsahariens qui débarquent sur les côtes européennes en passant par un deuxième pays de transit qui est la Libye, où ils s'exposent à des abus divers de la part des groupes armés, des milices, des réseaux de passeurs souvent liés à des réseaux criminels et/ou terroristes.

D'un autre côté, laTunisie fait toujours face aux départs irréguliers à l'étranger de milliers de ses jeunes, en quête d'emploi ou de meilleures conditions de vie ou de travail. Les quartiers populaires des grandes villes littorales, les zones rurales et les régions de l'intérieur continuent à alimenter les flux de départs irréguliers de jeunes tunisiens, alors que peu d'étudiants formés à l'étranger rentrent dans le pays au terme de leurs études. La situation de l'emploi ne s'est pas améliorée depuis la révolution, elle a même empiré, comme c'est le cas du contexte économique général marqué par le creusement des déficits, le recul des investissements directs étrangers et donc la baisse de la capacité des secteurs d'activité à générer de l'emploi. Le taux de chômage reste élevé $(15,5 \%)^{30}$.

À l'échelle régionale, la Tunisie est confrontée à la nécessité d'adapter sa gestion des questions migratoires qui ont changé de nature et d'ampleur depuis 2011, telles que l'asile, le séjour prolongé des Libyens sur le territoire tunisien, les relations migratoires avec I'Union européenne. Le projet de "Partenariat de Mobilité " $(\mathrm{PdM})^{31}$ avec I'UE est I'une des questions qui suscitent beaucoup de débats de la part des organisations de la société civile tunisienne, alors que le gouvernement tunisien, lui-même, cherche à limiter la réadmission aux ressortissants tunisiens qui font l'objet de mesures d'expulsion et pas les ressortissants de pays tiers, même s'il est prouvé qu'ils ont transité par la Tunisie.

La crise migratoire de l'année 2015, qui a vu affluer plus d'1 million de réfugiés vers l'Europe, a eu pour résultat un durcissement du discours de nombreux responsables européens sur la nécessité de restreindre l'accès des réfugiés au territoire européen ainsi que le renvoi des migrants dits "écono-

30 Surtout parmi les jeunes (entre 33 et $43 \%$ selon les tranches d'âge) et les diplômés de l'enseignement supérieur (32\%) (INS, 2013). Les jeunes femmes diplômées sont les plus touchées avec un taux de $42 \%$ en 2014. Mais si I'on prend en compte les jeunes qui sont en dehors de tout système éducatif, de formation ou d'emploi (NEET : Not in Education, in Employment or Training) (Banque Mondiale, 2014), cette catégorie représente la moitié $(50,4 \%)$ des femmes et le tiers $(33,4 \%)$ des hommes dans les zones rurales. Dans les zones urbaines, cette catégorie représente respectivement presque le tiers $(32,4 \%)$ des femmes et le cinquième $(20,3 \%)$ des hommes.

31 Les accords de "Partenariat de Mobilité " (PdM) sont signés entre I'Union européenne et des pays tiers, appartenant souvent au voisinage Est ou Sud de I'UE. Ils se distinguent par leur caractère juridiquement non contraignant. Quatre piliers forment ce type de partenariat : " L'organisation et la facilitation de l'immigration légale et de la mobilité ; la prévention et la réduction de l'immigration clandestine et de la traite des êtres humains ; la promotion de la protection internationale et le renforcement de la dimension extérieure de la politique d'asile et la maximisation de l'impact des migrations et de la mobilité sur le développement [...] ". Cf. Journal officiel de I'Union européenne (2012) COM(2011) 743 final, [en ligne], consulté le 05/01/2016. URL : http://eur-lex. europa.eu/legal-content/FR/TXT/?uri=CELEX:52012AE1057 
miques " qui se sont mêlés aux flux de réfugiés ${ }^{32}$. Comme les autres pays de transit, la Tunisie sera forcément confrontée aux retombées de la crise migratoire en Méditerranée sur les politiques migratoires européennes à travers leur implication dans ces politiques ${ }^{33}$ : externalisation, participation aux opérations de l'agence européenne Frontex en Méditerranée, accueil d'agents de Frontex sur le sol tunisien, transfert de données sur les passagers au départ, etc.

Le traitement de la mobilité des personnes et des migrations est devenu plus complexe avec l'émergence du terrorisme et des menaces aux frontières, surtout terrestres, de la Tunisie. Durant les dernières années, des milliers de Tunisiens ont rejoint les groupes djihadistes en Irak, en Syrie, au Sahara et en Libye, ou étaient impliqués dans des attentats ou des actes terroristes enTunisie. Les derniers attentats du Bardo (mars 2015), de Sousse (juin 2015) et de Tunis (24 novembre 2015) en sont une illustration parfaite. Des membres de ces groupes sont entrés à leur tour, et de manière irrégulière, par les frontières terrestres de la Tunisie, surtout à partir de la Libye. La mobilité des Tunisiens et des étrangers ne se pose plus uniquement en termes de contrôles aux frontières et de maitrise des flux, mais aussi en termes des risques que représentent ces passages au niveau sécuritaire, ce qui ouvre la voie au retour du traitement sécuritaire, voire même militariste, des flux migratoires.

\title{
Références bibliographiques
}

\begin{abstract}
Baba Wafa (2013) Investigation in first asylum country-Tunisia/Shousha Camp, KNOW RESET Research Report, 2013/02, Country of First Asylum Report, Florence, Migration Policy Centre and European Council on Refugees and Exiles, $32 \mathrm{p}$.
\end{abstract}

Banque Mondiale et Observatoire National de la Jeunesse (2014) Surmonter les obstacles à l'inclusion des Jeunes, Tunis, 127 p.

Ben Achour Souhayma (2011) Le droit tunisien face à la traite des personnes et au trafic des migrants, Note d'analyse et de synthèse, 2011/47, CARIM (Consortium euro-méditerranéen pour la recherche appliquée sur les migrations internationales), $32 \mathrm{p}$.

32 Ces responsables ont appelé au renforcement des contrôles des frontières extérieures de I'UE et au rétablissement des contrôles aux frontières intérieures à l'espace Schengen. Au plus haut de la crise de I'asile, en septembre et octobre 2015, la Hongrie a fermé ses frontières avec la Serbie, alors que la Croatie, I'Autriche et l'Allemagne ont rétabli les contrôles aux frontières internes. Les attentats de Paris du 13 novembre de la même année ont amené la France à faire de même. En l'espace de deux semaines, deux sommets ont été organisés avec les pays d'origine ou de transit pour la maîtrise des flux de réfugiés et de migrants. Le premier sommet (les 11 et 12 novembre) a réuni à Malte les pays membres de I'UE avec les pays africains, alors que le deuxième sommet (le 29 novembre) a réuni la Turquie avec les pays membres. Cf. Le Monde (2015) L'Europe cherche à fermer la route des migrants d'Afrique et de Turquie, [en ligne], consulté le 05/01/2016. URL : http://www.lemonde.fr/europe/article/2015/11/11/plusieurs-migrantsmeurent-dans-le-naufrage-d-une-embarcation-au-large-de-la-turquie_4807126_3214. html?xtmc=I_europe_cherche_a_fermer_la_route_des_migrants_d_afrique_et_de_ turquie\&xtcr=1

33 Le nouvel "Agenda européen pour la migration " prévoit d'impliquer les pays tiers et de transit (telle la Tunisie) dans la mise en œuvre de cette politique. Cf. European Commission (2015) European Agenda on Migration, [en ligne], consulté le 05/01/2016. URL : http://ec.europa.eu/dgs/home-affairs/what-we-do/policies/european-agenda-migration/index_en.htm 
Ben Jémia Monia et Ben Achour Souhayma (2015) Plaidoyer pour une réforme des lois relatives aux migrants, aux étrangers et à la nationalité en Tunisie, Sousse, REMDH et CeTuMA, 50 p.

Ben Jémia Monia et Ben Achour Souhayma (2011) Guerre en Libye : la situation des migrants et des réfugiés en Tunisie, Note d'analyse et de synthèse - module juridique, Florence, CARIM, $22 \mathrm{p}$.

Boubakri Hassan (2014). Labour migration management in contemporary Tunisia and Egypt, Rapport non publié, Cairo, CMRS/American University of Cairo, 30 p.

Boubakri Hassan (2013a) Les migrations en Tunisie après la révolution. Gestion de l'asile et enjeux internationaux, Confluences Méditerranée, 87, pp. 31-46.

Boubakri Hassan (2013b) Revolution and international migration in Tunisia, MPC Research Report, 2013/04, Florence, Migration Policy Centre, 34 p., [online]. URL: http://www.migrationpolicycentre.eu/docs/MPC-RR-2013-04.pdf (eng); http:// www.migrationpolicycentre.eu/docs/MPC-RR-2013-01.pdf (fr).

Boubakri Hassan (2009) L'administration des migrations irrégulières par l'État tunisien : dispositifs règlementaires et relations avec l'Europe, in Abdelkhaleq Berramdane et Jean Rossetto, La politique européenne d'immigration, Paris, Karthala, pp. 285-309.

Boubakri Hassan (2006a) Le Maghreb et les migrations de transit : le piège ?, Migrations et Société, 107, pp. 85-104.

Boubakri Hassan (2006b) L'autre face de la Jeffara (Sud-Est tunisien) : mobilité transfrontalière, migration internationale et dynamiques territoriales, in Didier Genin et Henri Guillaume Éds., Entre désertification, et développement : la Jeffara tunisienne, Tunis et Paris, Cérès éditions et IRD Édition, pp. 197-213.

Boubakri Hassan et Nouri Habiba (2009) Migrations, transformations sociales et recompositions des territoires dans la Jeffara (Sud-Est de la Tunisie), in Pierre Bonte, Mohamed Elloumi, Henri Guillaume et Mohamed Madhi Dirs., Développement rural, environnement et enjeux territoriaux : regards croisés Oriental marocain et Sud-Est tunisien, Tunis, Cérès éditions et IRD Édition, pp. 113-142.

Boubakri Hassan et Potot Swanie (2012) De l'élan citoyen à la mise en place d'une politique migratoire en Tunisie : I'accueil des réfugiés de Libye en 2011, Migrations Société, 24 (143), pp. 121-137.

Boubakri Hassan et Potot Swanie (2011) Exode et migrations en Tunisie : quand la société civile se réveille, Blog Médiapart et Lettre de I'IRMC, 6, 2 p., [en ligne]. URL : http://blogs.mediapart.fr/blog/swpotot/100311/exode-et-migrations-entunisie-quand-la-societe-civile-se-reveille

Chouat Mokhtar et Liteyem Borniya (2011) Migrations et asile dans le Sud-Est tunisien, Mémoire de fin d'étude (en langue arabe), non publié, Université de Sousse, $35 \mathrm{p}$.

Fargues Philippe and Fandrich Christine (2012) Migration after the Arab Spring, MPC Research Report 2012/09, Florence, Migration Policy Centre, 22 p.

Frontex (2015) Annual_Risk_Analysis 2014, 72 p.

Frontex (2014) Annual_Risk_Analysis 2013, 84 p.

Frontex (2013) Annual_Risk_Analysis 2012, 69 p.

Frontex (2012) Annual_Risk_Analysis 2011, 64 p. 
Frontex (2011) Annual_Risk_Analysis 2010, 36 p.

Institut National de la Statistique (INS) (2015) Recensement général de la Population et de I'Habitat de 2014. Résultats préliminaires, Tunis, INS, 36 p.

Institut National de la Statistique (INS) (2013a) Enquête Population Emploi 2012, Tunis, INS, $223 \mathrm{p}$.

Institut National de la Statistique (INS) (2013b) Annuaire statistique de la Tunisie 2008-2012, Tunis, INS, 357 p.

International Crisis Group (ICG) (2013) LaTunisie des frontières : Jihad et contrebande, Rapport Moyen-Orient/Afrique du Nord, 148, 50 p.

Natter Katharina (2014) Fifty years of Maghreb emigration. How states shaped Algerian, Moroccan and Tunisian emigration, Working Papers, 95, Oxford, International Migration Institute, University of Oxford, $35 \mathrm{p}$.

Organisation Internationale pour les Migrations (OIM) (2014) Étude exploratoire sur la traite des personnes en Tunisie, Tunis, IOM, $106 \mathrm{p}$.

Organisation Internationale pour les Migrations (OIM) (2011) IOM response to the Libyan Crisis, External Situation Report, 3 p., [online]. URL: https://www.iom.int/ jahia/webdav/shared/shared/mainsite/media/docs/reports/IOM-sitrep-MENA.pdf

REMDH et CeTuMA (2015) Plaidoyer pour une réforme des lois relatives aux migrants, aux étrangers et à la nationalité en Tunisie, $50 \mathrm{p}$.

Secrétariat d'État à la Migration et aux Tunisiens à I'Étranger (SEMTE) (2013) Stratégie Nationale de l'Émigration, $36 \mathrm{p}$.

UNHCR (2015) Tunisia Fact Sheet, July 2015, 3 p.

UNHCR (2014) Tunisia Fact Sheet, September 2014, 2 p.

UNHCR (2013) Bulletin d'information, Tunis, Mars 2013, 2 p.

UNICEF and UNFPA (2014) Impact de la Migration sur les familles des migrants présentes en Tunisie, Tunis, $263 \mathrm{p}$. 


\section{Hassan Boubakri}

\section{Migration et asile en Tunisie depuis 2011 : vers de nouvelles figures migratoires?}

LaTunisie, premier pays arabe à avoir connu un soulèvement populaire en 2011, est passée par une expérience migratoire que l'on peut qualifier d'inédite pour un pays traditionnel d'émigration. Avant 2011, le pays accueillait officiellement une centaine de réfugiés reconnus par le HCR et voyait quelques milliers de migrants irréguliers (Tunisiens et étrangers) partir ou transiter par son territoire pour traverser la mer en direction des côtes italiennes. Mais depuis 2011, le nombre d'étrangers fuyant les guerres civiles et l'instabilité dans leurs pays d'origine, ainsi que la pauvreté et le chômage, n'a cessé d'augmenter. Si les premières vagues d'exil ont pris fin en 2011, des centaines de milliers de ressortissants libyens continuent à entrer en Tunisie pour des séjours prolongés et renouvelés en raison de la reprise de la guerre civile depuis 2014 et la désorganisation des services publics. Le voisinage et la libre circulation des personnes entre les deux pays rend difficile la qualification de la présence des Libyens en Tunisie : sont-ils touristes ou réfugiés ? La guerre civile syrienne a également forcé quelques milliers de réfugiés, une infime minorité, à choisir la Tunisie comme pays d'asile ou de transit pour l'Europe. LaTunisie continue à abriter une immigration d'origine subsaharienne alimentée par les étudiants, les migrants économiques, mais aussi par les réfugiés fuyant les anciens et les nouveaux foyers de conflits sur le continent. Cet article traite de la manière dont ce pays accueille et gère ces différentes formes de migration.

\section{Migration and Asylum in Tunisia since 2011: Towards a New Migration Profile?}

Tunisia, the first Arab country to have known a popular uprising, went through a migratory experience that we can qualify as the "novel" for a traditional issuing country. Before 2011, it was home to no more than a hundred refugees, and saw some thousands irregular migrants (Tunisian and foreign) lifting or crossing through its territory to reach, by sea, the Italian coast. But since 2011, the number of foreigners fleeing civil wars and instability in their country of origin, but also poverty and unemployment, has been rising. If the first wave of exile ended in 2011, hundreds of thousands of Libyan nationals continue to enter Tunisia for extended stays and renewed due to the resumption of civil war since 2014 and the disruption of public services. The neighborhood and the free movement of persons between both countries make it difficult the qualification of the presence of Libyan nationals in Tunisia: are they tourists or refugees? Syrian civil war has also forced few thousands of refugees to reach Tunisia as country of asylum or to transit for Europe. Tunisia continues to be a destination of sub-Saharan immigration fueled by students, economic migrants, and also by refugees fleeing old and new homes of conflicts on the continent. The article examines the ways in which this country hosts and manages different forms of migration. 


\section{..’⿳亠丷冖̆ Migración y asilo en Túnez desde 2011: ¿hacia un nuevo perfil de la migración?}

Túnez, el primer país árabe que ha experimentado un levantamiento popular en 2011, ha pasado por una experiencia migratoria que se puede calificar de novedosa para un país de tradición emigratoria. Antes de 2011, el país acogía oficialmente a un centenar de refugiados reconocidos por ACNUR y veía a varios miles de migrantes irregulares (tunecinos y extranjeros) salir o estar de tránsito por su territorio para cruzar el mar hacia las costas italianas. Pero desde 2011, el número de extranjeros que huyen de guerras civiles y de la inestabilidad en sus países de origen, aunque también de la pobreza y del desempleo, ha ido en constante aumento. Si las primeras olas de exilio terminaron en 2011, cientos de miles de ciudadanos libios siguen entrando en Túnez para estancias largas y renovadas, debido a la reanudación de la guerra civil desde 2014 y la interrupción de los servicios públicos. La frontera común y la libre circulación de personas entre los dos países hace difícil la calificación de la presencia de ciudadanos libios enTúnez: ¿son turistas o refugiados? La guerra civil de Siria también ha obligado a miles de refugiados de ese país a elegir Túnez como país de asilo o de tránsito hacia Europa. Túnez sigue albergando una inmigración de origen subsahariana alimentada por los estudiantes, los migrantes económicos, pero también por los refugiados que huyen de antiguos y nuevos focos de conflicto en el continente. Este trabajo trata la forma en la que este país acoge y gestiona estas diferentes formas de migración. 